\title{
Mathematical, numerical and experimental analysis of the swirling flow at a Kaplan runner outlet
}

\author{
S Muntean ${ }^{1,5}$, T Ciocan ${ }^{2}$, R F Susan-Resiga ${ }^{2}$, M Cervantes $^{3}$ and H Nilsson ${ }^{4}$ \\ ${ }^{1}$ Center for Advanced Research in Engineering Science, Romanian Academy - \\ Timişoara Branch, Bv. Mihai Viteazu, No.24, Timisoara, RO-300223, Romania \\ ${ }^{2}$ Hydraulic Machinery Department, Politehnica University of Timişoara, Bvd. Mihai \\ Viteazu, No.1, Timişoara, RO-300222, Romania \\ 3 Department of Engineering Science and Mathematics, Luleå University of \\ Technology, Myntvägen No. 8, SE-97 452 Luleå, Sweden \\ ${ }^{4}$ Department of Applied Mechanics, Chalmers University of Technology, SE-412 96 \\ Gothenburg, Sweden \\ E-mail: seby@acad-tim.tm.edu.ro
}

\begin{abstract}
The paper presents a novel mathematical model for a-priori computation of the swirling flow at Kaplan runners outlet. The model is an extension of the initial version developed by Susan-Resiga et al [1], to include the contributions of non-negligible radial velocity and of the variable rothalpy. Simple analytical expressions are derived for these additional data from three-dimensional numerical simulations of the Kaplan turbine. The final results, i.e. velocity components profiles, are validated against experimental data at two operating points, with the same Kaplan runner blades opening, but variable discharge.
\end{abstract}

\section{Introduction}

The main advantages of Kaplan turbines are the wide ranges of gate opening and heads which can be guaranteed and because of the vertical arrangement, the possibility of installing high capacity units under low heads. It should be noted that the mechanical complexity of adjustable blade turbines is counterbalanced by the flat efficiency diagrams and stable hydraulic behavior in a wide range of discharges and heads [2].

The hydrodynamics of Kaplan turbines is a more than a century old subject that benefits from a large body of both theoretical and experimental investigations (Anton [3], Krishna [4]). Traditionally, the main subject of these studies has been the turbine runner, with the development of powerful and reliable tools for designing the runner blades. When developing a new runner for existing turbines refurbishment, usually the draft tube remains unchanged. As a result, the new runner should be the best match for the existing draft tube within a wide operating range. In order to achieve this goal, there are two main approaches: i) develop several runner geometries using geometric parameterization and use a-posteriori analysis (usually numerical) to assess the turbine performance; ii) optimize the swirling flow at the draft tube inlet, then design the runner accordingly. The second approach is currently used for swirl optimization at the design operating point. However, since modern hydraulic

\footnotetext{
${ }^{5}$ To whom any correspondence should be addressed
} 
turbines should operate within a wide range it is essential to optimize the swirl at runner outlet over multiple operating regimes in connection with the existing draft tube.

The Turbine-99 (T-99) workshops have been organized in order to assess the capacity of simulation tools for calculation of flow in hydraulic draft tubes [5-7]. The model turbine studied in at T-99 is a 1:11 scale of a power station, and the Kaplan runner model has a diameter of $0.5 \mathrm{~m}$, Fig. 1 . The Reynolds number based on runner diameter and average discharge velocity is $1.75 \times 10^{6}$.
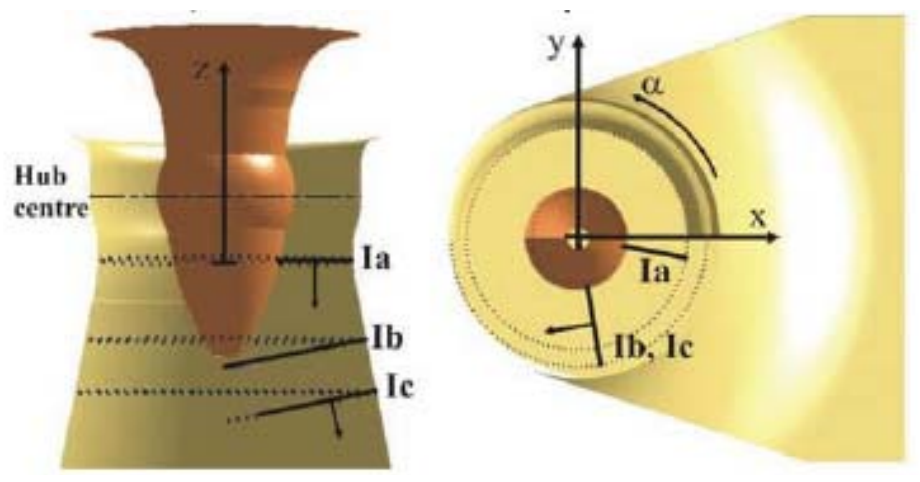

Figure 1. T-99 cross section (left) and top view (right), [8]

The T-99 test case consists of data for two operating points. The first operating point is on-cam, i.e. top-point ( $\mathrm{T}$ case) on the propeller curve denoted BEP, with discharge $0.522 \mathrm{~m}^{3} / \mathrm{s}$. The second operating point is off-cam, i.e the right-leg ( $\mathrm{R}$ case) on the propeller curve labeled FL, with discharge $0.554 \mathrm{~m}^{3} / \mathrm{s}$. Both operating regimes have the same head, $4.5 \mathrm{~m}$, and runner speed, $595 \mathrm{rpm}$, for the Kaplan turbine model. Two velocity components measured with LDV system just downstream of the runner (section Ia) are available [8].

The main purpose of the paper is to introduce and validate a quasi-analytical model for computing the axi-symmetric velocity field at the outlet of the Kaplan runner, for variable operating regimes, without knowing the runner geometry. Such a model is useful in the preliminary design stages, for optimizing the swirl ingested by the draft tube within an operating range.

Section 2 presents the mathematical model for swirling flow at runner outlet of hydraulic turbines as well as the variational formulation with associated constrains. Next, the swirl-free velocity for the T99 Kaplan runner outlet is computed in Section 3, while the rothalpy and radial velocity distributions are evaluated in Sections 4 and 5, respectively. The swirling flow at the T99 Kaplan runner outlet is computed using the mathematical model developed in this paper. The analytical profiles are validated against experimental data and numerical results in Section 6. The conclusions are drawn in the last section.

\section{Mathematical model for swirling flow at a runner outlet}

The mathematical model for the swirling flow downstream a Kaplan runner is derived under a set of simplifying assumptions, based on a two-dimensional model of inviscid steady axisymmetric swirling flows in turbomachines. We present in this section the mathematical derivation as well as the numerical algorithm for solving the boundary-value problem.

2.1. Axisymmetric steady swirling flow in turbomachines

The simplified mathematical model used in this paper is derived from a more general model for axisymmetric swirling flows in turbomachines, based on the steady Euler equation written in a coordinate system rotating with the runner,

$$
(\nabla \times \boldsymbol{V}) \times \boldsymbol{W}=-\nabla I+\boldsymbol{F}_{B},
$$


where $\boldsymbol{V}$ is the absolute velocity, $\boldsymbol{W}$ the relative velocity, with axial, radial and circumferential components $\left(W_{z}, W_{r}, W_{\theta}\right)=\left(V_{z}, V_{r}, V_{\theta}-\Omega R\right)$, respectively, $I \equiv P / \rho+V^{2} / 2-\Omega R V_{\theta}$ is the relative specific energy or rothalpy, $\boldsymbol{F}_{B}$ is a fictitious body force acceleration that replaces the effect of the blades on turning the flow, $\Omega$ is the runner angular speed, and $(Z, R, \theta)$ are the cylindrical coordinates. A typical hub-to-shroud streamsurface in the bladed region can be geometrically described as

$$
\Theta(Z, R, \theta)=\theta-\varphi(Z, R)=\text { constant },
$$

where $\varphi(Z, R)$ is the so-called blade wrapping angle. The $\Theta=$ constant streamsurface for the relative flow is associated with the blade shape for an infinite number of blades, infinitely thin. The blade body force acceleration $\boldsymbol{F}_{B}$ is normal to such a streamsurface, along the unit normal vector

$$
\boldsymbol{n}_{B}=\frac{\nabla \Theta}{|\nabla \Theta|}=\left(\boldsymbol{e}_{\theta}-\frac{R \partial \varphi}{\partial Z} \boldsymbol{e}_{z}-\frac{R \partial \varphi}{\partial R} \boldsymbol{e}_{r}\right) / \sqrt{1+\left(\frac{R \partial \varphi}{\partial Z}\right)^{2}+\left(\frac{R \partial \varphi}{\partial R}\right)^{2}}
$$

On the $\Theta=$ constant streamsurface we have two tangent unit vectors, $\boldsymbol{\tau}_{1}=\boldsymbol{W} / \boldsymbol{W}$, along the streamline in relative flow, and $\boldsymbol{\tau}_{2}=\boldsymbol{n}_{B} \times \boldsymbol{\tau}_{1}$. The flow tangency condition can be simply written as $\boldsymbol{\tau}_{1} \cdot \boldsymbol{n}_{B}=0$, or

$$
V_{z} \frac{\partial \varphi}{\partial Z}+V_{r} \frac{\partial \varphi}{\partial R}=\frac{V_{\theta}}{R}-\Omega
$$

When projecting Eq.(1) along the $\boldsymbol{\tau}_{2}$ direction, we obtain the so-called principal equation for axisymmetric turbomachinery swirling flow,

$$
\begin{aligned}
& \frac{\partial V_{r}}{\partial Z}-\frac{\partial V_{z}}{\partial R}=\frac{\partial \varphi}{\partial Z} \frac{\partial\left(R V_{\theta}\right)}{\partial R}-\frac{\partial \varphi}{\partial R} \frac{\partial\left(R V_{\theta}\right)}{\partial Z} \\
& +\frac{1}{W^{2}}\left[\left(W_{r}+\frac{\partial \varphi}{\partial R} R W_{\theta}\right) \frac{\partial I}{\partial Z}-\left(W_{z}+\frac{\partial \varphi}{\partial Z} R W_{\theta}\right) \frac{\partial I}{\partial R}\right]
\end{aligned}
$$

2.1.1. Streamfunction formulation. Eq.(5) is more conveniently expressed using the Stokes' streamfunction for incompressible axisymmetric flows, $\Psi$, defined as

$$
V_{z}=W_{z}=\frac{1}{R} \frac{\partial \Psi}{\partial R} \text { and } V_{r}=W_{r}=-\frac{1}{R} \frac{\partial \Psi}{\partial Z},
$$

thus automatically satisfying the continuity equation $\nabla \cdot \boldsymbol{V}=0$. Moreover, projecting Eq.(1) along the direction of $\tau_{1}$ gives the Bernoulli's theorem in relative flow, $\boldsymbol{W} \cdot \nabla I=0$, i.e. the rothalpy is a function only of the streamfunction, $I(\Psi)$. As a result, the last term in the right-hand side of Eq.(5) simply becomes $-R(\mathrm{~d} I / \mathrm{d} \Psi)$, i.e.

$$
-\frac{\partial}{\partial Z}\left(\frac{1}{R} \frac{\partial \Psi}{\partial Z}\right)-\frac{\partial}{\partial R}\left(\frac{1}{R} \frac{\partial \Psi}{\partial R}\right)=\frac{\partial \varphi}{\partial Z} \frac{\partial\left(R V_{\theta}\right)}{\partial R}-\frac{\partial \varphi}{\partial R} \frac{\partial\left(R V_{\theta}\right)}{\partial Z}-R \frac{\mathrm{d} I}{\mathrm{~d} \Psi}
$$

2.2. Axisymmetric steady swirling flow at a runner outlet

The principal equation (7), together with the flow tangency condition (4), can be used either in the design mode (given $R V_{\theta}$, find $\Psi$ and $\varphi$ ) or in the analysis mode (given $\varphi$, find $\Psi$ and $R V_{\theta}$ ). In this paper we consider the analysis mode, focused on the swirling flow at runner outlet.

2.2.1. The swirl-free velocity. For vanishing radial velocity, the flow tangency condition from Eq.(4) can be written using the so-called swirl-free velocity, $V_{\text {sf }}$, as defined in Eq.(8) below. As shown by the velocity triangle at the runner outlet, Fig. 2 , the swirl-free velocity is the value of the axial velocity which corresponds to vanishing circumferential velocity at each radius. 


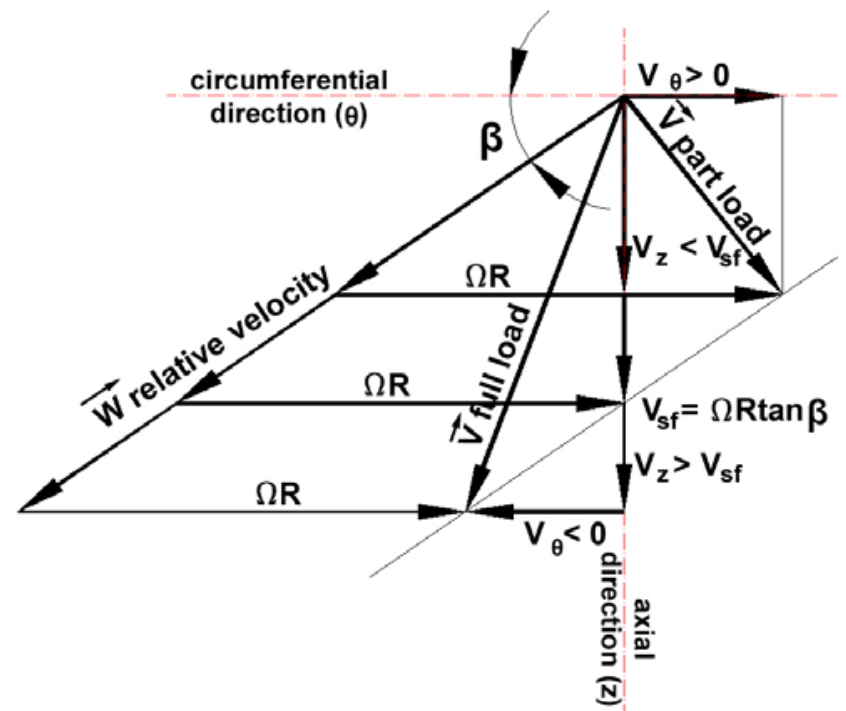

As a matter of fact, the swirl-free velocity profile $V_{\mathrm{sf}}(R)$ is a convenient way to describe the blade geometry at the trailing edge, instead of the more traditional relative flow angle. In a first approximation, when no severe flow detachment is present, $V_{\text {sf }}(R)$ does not depend on the operating point.

$$
\frac{\partial \varphi}{\partial Z}=-\frac{\Omega}{V_{\mathrm{sf}}}, \text { where } V_{\mathrm{sf}} \equiv \frac{\Omega R V_{z}}{\Omega R-V_{\theta}}
$$

Figure 2. Velocity triangles at runner outlet.

2.2.2. One-dimensional swirling flow model at runner outlet. Assuming that the local flow changes in the axial direction are negligible (parallel or columnar swirling flow), one can simplify Eq.(7), using Eq.(8), into a one-dimensional swirling flow model,

$$
4 \frac{\mathrm{d}^{2} \Psi}{\mathrm{d} Y^{2}}=\frac{2}{V_{\mathrm{sf}}} \frac{\mathrm{d}\left(\Omega R V_{\theta}\right)}{\mathrm{d} Y}+\frac{\mathrm{d} I}{\mathrm{~d} \Psi}, Y \equiv R^{2}
$$

where we have introduced the modified radial coordinate $Y$. It is convenient to re-write Eq.(9) into a dimensionless form by considering a reference length $R_{\text {ref }}$ and a reference velocity $V_{\text {ref }}=\Omega R_{\text {ref }}$,

$$
4 \frac{\mathrm{d}^{2} \psi}{\mathrm{d} y^{2}}=\frac{2}{v_{\mathrm{sf}}} \frac{\mathrm{d} c}{\mathrm{~d} y}+\frac{\mathrm{d} i}{\mathrm{~d} \psi}, \text { where } c \equiv r v_{\theta}=y\left(1-\frac{v_{z}}{v_{\mathrm{sf}}}\right) \text { and } \frac{\mathrm{d} \psi}{\mathrm{d} y}=\frac{v_{z}}{2}
$$

Given the swirl-free velocity profile $v_{\text {sf }}(y)$ and the rothalpy variation $i(\psi)$, Eq.(10) with additional conditions, allows the computation of the axial velocity profile. The circumferential velocity immediately follows as $v_{\theta}=r\left(1-v_{z} / v_{\text {sf }}\right)$.

\subsubsection{Variational formulation for the constrained swirling flow problem.}

The differential model from Eq.(10) has an equivalent variational formulation which is more suitable for developing robust numerical algorithms. Let us consider the functional $\mathrm{F}(\psi)$ defined as,

$$
\mathrm{F}(\psi)=\int_{y_{\text {wub }}}^{y_{\text {tip }}}\left[2\left(\frac{\mathrm{d} \psi}{\mathrm{d} y}\right)^{2}-\frac{c^{2}}{2 y}+c+i(\psi)\right] \mathrm{d} y
$$

In order to find the solution that cancels the Fréchet differential of this functional, $\lim _{\varepsilon \rightarrow 0}[\mathrm{~F}(\psi+\varepsilon \eta)-\mathrm{F}(\psi)] / \varepsilon=0$, we consider an arbitrary perturbation of the streamfunction, $\eta(y)$, which vanishes at the boundaries. After integrating by parts we obtain

$$
\int_{y_{\text {hub }}}^{y_{\text {tip }}} \eta\left[-4 \frac{\mathrm{d}^{2} \psi}{\mathrm{d} y^{2}}+\frac{2}{v_{\text {sf }}} \frac{\mathrm{d} c}{\mathrm{~d} y}+\frac{\mathrm{d} i(\psi)}{\mathrm{d} \psi}\right] \mathrm{d} y+\left.\left(4 \eta \frac{\mathrm{d} \psi}{\mathrm{d} y}\right)\right|_{y_{\text {tub }}} ^{y_{\text {tip }}}=0 .
$$

Since $\eta(y)$ is arbitrary and vanishes at the boundaries, it follows that it is the solution of the differential Eq.(10) that corresponds to an extremum of the functional from Eq.(11). 
We can now state the full variational problem for the swirling flow downstream the Kaplan runner: given the dimensionless volumetric flow rate, $q$, the dimensionless flux of moment of momentum, $m$, the rothalpy variation, $i(\psi)$, and the swirl-free velocity profile, $v_{\mathrm{sf}}(y)$, find the axial velocity profile $v_{z}(y)$ which minimizes the functional with constraints,

$$
\begin{gathered}
\mathrm{F}\left(v_{z}\right)=\int_{y_{\text {tub }}}^{y_{\text {tip }}}\left[\frac{v_{m}^{2}}{2}+\frac{y}{2}\left(1-\frac{v_{z}^{2}}{v_{\mathrm{sf}}^{2}}\right)+i(\psi)\right] \mathrm{d} y=\min , \\
\int_{y_{\text {hub }}}^{y_{\text {tip }}} v_{z} \mathrm{~d} y=q, \\
\int_{y_{\text {hub }}}^{y_{\text {tip }}} c v_{z} \mathrm{~d} y=\int_{y_{\text {hub }}}^{y_{\text {tip }}} v_{z}\left(1-\frac{v_{z}}{v_{\text {sf }}}\right) y \mathrm{~d} y=m .
\end{gathered}
$$

The first term in the functional $\mathrm{F}\left(v_{z}\right)$ was modified to accommodate a contribution of the radial velocity, by replacing $v_{z}^{2} / 2$ with $v_{m}^{2} / 2=\left(v_{z}^{2}+v_{r}^{2}\right) / 2$, consistent with functional form for two axisymmetric swirling flows, Wang and Rusak [9]. Note that the integral constraints (12b) and (12c) correspond to the two integral quantities that characterize the swirling flow downstream a turbine runner.

2.3. Fourier-Bessel series approximation for the axial velocity

In order to solve numerically the constrained variational problem given by Eqs. (12) we need an approximation for the unknown function $v_{z}$. We have introduced such an approximation in [1], using a Fourier-Bessel series as follows,

$$
\begin{aligned}
& v_{z}\left(y ; a_{1}, a_{2}, \ldots, a_{N}, y_{\text {hub }}, y_{\text {tip }}, q\right)=\frac{q}{y_{\text {tip }}-y_{\text {hub }}}+\sum_{i=1}^{N} a_{i} \mu_{i}\left(y ; y_{\text {hub }}, y_{\text {tip }}\right) \\
& \mu_{i}\left(y ; y_{\text {hub }}, y_{\text {tip }}\right)=J_{0}\left(\lambda_{i} \sqrt{\frac{y}{y_{\text {tip }}}}\right)+\frac{2 \sqrt{y_{\text {hub }} y_{\text {tip }}}}{y_{\text {tip }}-y_{\text {hub }}} \frac{1}{\lambda_{i}} J_{1}\left(\lambda_{i} \sqrt{\frac{y_{\text {hub }}}{y_{\text {tip }}}}\right),
\end{aligned}
$$

where $\lambda_{i}$ are the zeros of the $J_{1}$ Bessel function, i.e. $J_{1}\left(\lambda_{i}\right)=0$. The series approximation for the axial velocity automatically satisfies the discharge constraint (12b). The streamfunction approximation immediately follows as,

$$
\begin{aligned}
& \psi\left(y ; a_{1}, a_{2}, \ldots, a_{N}, y_{\text {hub }}, y_{\text {tip }}, q\right)=\frac{q}{2} \frac{y-y_{\text {hub }}}{y_{\text {tip }}-y_{\text {hub }}}+\sum_{i=1}^{N} a_{i} \eta_{i}\left(y ; y_{\text {hub }}, y_{\text {tip }}\right) \\
& \eta_{i}\left(y ; y_{\text {hub }}, y_{\text {tip }}\right)=\frac{\sqrt{y y_{\text {tip }}}}{\lambda_{i}} J_{1}\left(\lambda_{i} \sqrt{\frac{y}{y_{\text {tip }}}}\right)-\frac{y_{\text {tip }}-y}{y_{\text {tip }}-y_{\text {hub }}} \frac{\sqrt{y_{\text {hub }} y_{\text {tip }}}}{\lambda_{i}} J_{1}\left(\lambda_{i} \sqrt{\frac{y_{\text {hub }}}{y_{\text {tip }}}}\right)
\end{aligned}
$$

We obviously have $\psi\left(y_{\text {hub }}\right)=0$ and $\psi\left(y_{\text {tip }}\right)=q / 2$, thus the discharge constraint (12b) is satisfied, as expected. Both approximations (13) and (14) have leading terms corresponding to a constant axial velocity profile, which are corrected with a linear combination of modes with vanishing discharge.

Inserting this approximation in the functional (12a), together with the integral constraint (12c), leads to a problem for the unknown mode amplitudes $a_{1}, a_{2}, \ldots, a_{N}$.

\section{Swirl-free velocity for a Kaplan runner outlet}

The swirl-free velocity is computed using data for axial and circumferential velocity components, respectively, using the definition from Eq.(8). 


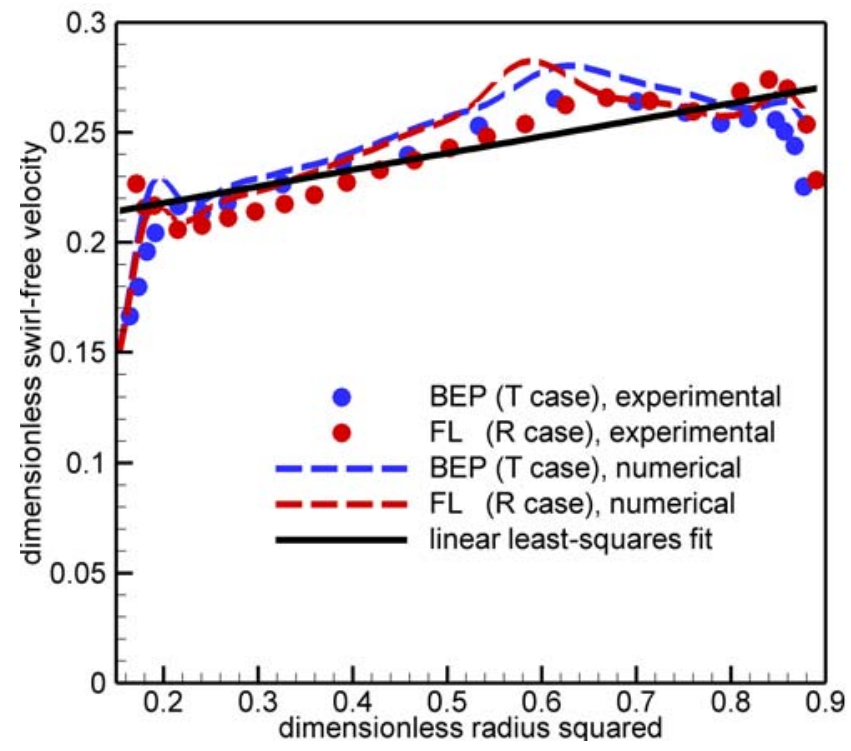

Figure 3. Swirl-free velocity profile.

\section{Rothalpy variation for Kaplan turbine}

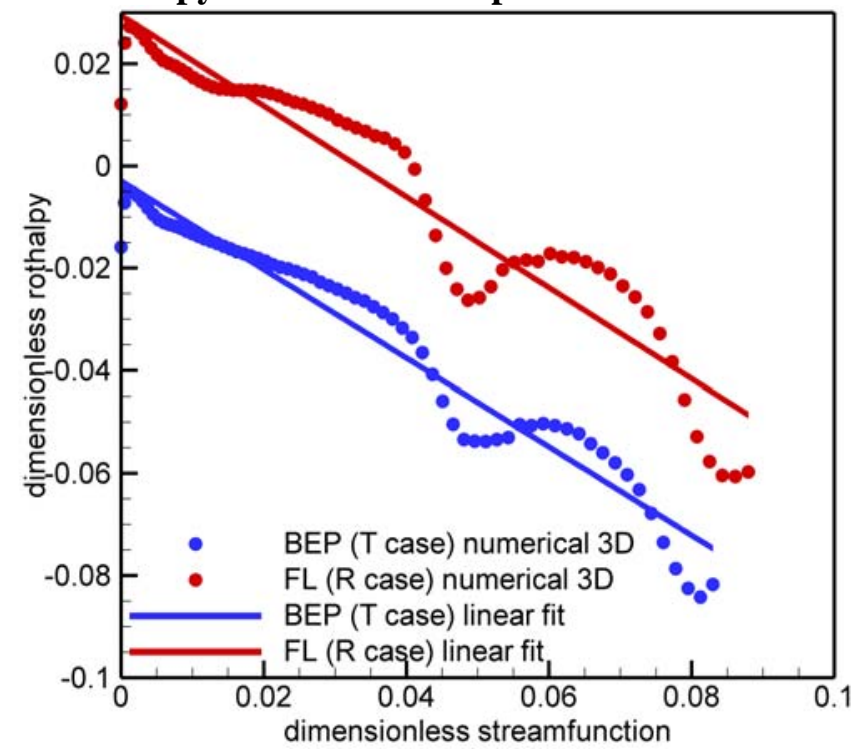

Figure 4. Rothalpy variation with the streamfunction
For example, Fig. 3 shows the dimensionless $v_{\mathrm{sf}}$ computed from experimental data (circles) or numerical results (dashed lines) for the Kaplan turbine investigated in the present paper. As we have found for Francis turbines, [1], a linear fit with respect to the dimensionless radius squared is accurate enough to represent the swirl-free velocity within our simplified model, i.e.

$$
v_{\mathrm{sf}}(y)=v_{\mathrm{sf}}^{(0)}+v_{\mathrm{sf}}^{(1)} y \text {. }
$$

The fit coefficients are found from experimental data for the Kaplan runner investigated in the paper as $v_{\mathrm{sf}}^{(0)}=0.2027$ and $v_{\mathrm{sf}}^{(1)}=0.0756$, respectively.

\section{The radial velocity at a Kaplan runner outlet}

The radial component of the velocity at the runner outlet plays an important role in achieving accurate numerical results for the draft tube flow field. A first approximation of the radial velocity component was developed for the T-99 test case by Bergström [10] assuming a linear distribution of the flow angle. Cervantes and Gustavsson [11] further analyzed this radial velocity component an attempt to develop a more rigorous approach. On the other hand, when computing the radial velocity one needs to evaluate the axial derivative of the streamfunction, as shown in Eq.(6). It is clear that within the present model, which considers the flow only on a survey cross-section it is impossible to completely evaluate axial derivatives. However, an approximation for the radial velocity can be found if we consider only the gradients of the hub and shroud walls, $d r_{\text {hub }} / d z$ and $d r_{\text {tip }} / d z$, respectively. Using the streamfunction expression, Eq.(14), and taking into account the expression for the axial velocity, Eq.(13), one can show that,

The dimensionless rothalpy is computed from the numerical velocity and pressure fields, and plotted versus the streamfunction in Fig.3. Simple linear fits are considered, given the simplified assumptions of our swirl model, resulting at BEP ( $\mathrm{T}$ case) $i(\psi)=-0.002959-0.86488 \psi$, and at FL $(\mathrm{R}$ case) $i(\psi)=0.029479-0.88227 \psi$, respectively. Note that the rothalpy is defined up to an arbitrary additive constant, and as a result only the gradients of the above linear fits are relevant for the minimization of the functional (12a). The rothalpy variation is due to the fact that the straight guide vanes are located in the flow turning region for low head turbines, and as expected the rothalpy decreases from hub to shroud. 


$$
v_{r}(r)=-\frac{1}{r} \frac{\partial \psi}{\partial z} \cong v_{z}(r)\left[\frac{r_{\text {tip }}^{2}-r^{2}}{r_{\text {tip }}^{2}-r_{\text {hub }}^{2}} \frac{r_{\text {hub }}}{r} \frac{\mathrm{d} r_{\text {hub }}}{\mathrm{d} z}+\frac{r^{2}-r_{\text {hub }}^{2}}{r_{\text {tip }}^{2}-r_{\text {hub }}^{2}} \frac{r_{\text {tip }}}{r} \frac{\mathrm{d} r_{\text {tip }}}{\mathrm{d} z}\right] .
$$

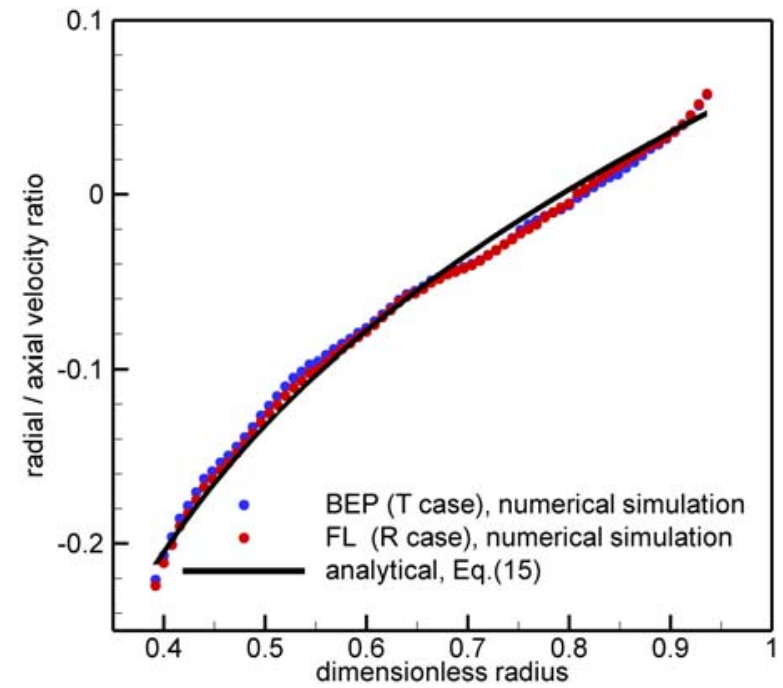

In our case, we have $d r_{\text {hub }} / d z=-0.212$ and $d r_{\text {tip }} / d z=0.0466$ from the geometry shown in Fig.1. In order to check the accuracy of Eq.(16) we plot the ratio $v_{r}(r) / v_{z}(r)$ computed from numerical simulations of the full 3D flow in the Kaplan turbine runner, Fig. 5. One can see that the simple analytical expression from Eq.(16) is in excellent agreement with numerical results. Such an agreement is to be expected as long as the flow tangency conditions at the hub and shroud, respectively, are satisfied. When the flow detaches from the hub, as it is the case for partial discharge regimes, the simple analytical approximation is no longer valid.

Figure 5. Radial / axial velocity components ratio.

\section{Swirling flow at a Kaplan runner outlet}

The annular survey section shown in Fig. 1 has the hub and tip radii $R_{\text {hub }}=0.098 \mathrm{~m}$ and $R_{\text {tip }}=0.236 \mathrm{~m}$, respectively, with the corresponding modified dimensionless radial coordinate values $y_{\text {hub }}=0.154$ and $y_{\text {tip }}=0.891$. The best efficiency operating point ( $\mathrm{T}$ case) is defined by the dimensionless discharge $q_{\mathrm{BEP}}=0.1567$ and the dimensionless flux of moment of momentum $m_{\mathrm{BEP}}=0.8080 \mathrm{E}-2$, respectively. The corresponding values for the full load point (R case) are $q_{\mathrm{FL}}=0.1693$ and $m_{\mathrm{FL}}=0.3831 \mathrm{E}-2$. Note that the above values have been determined by numerical integration of the experimental data, and as expected the results obtained with the mathematical model presented in section 2 are in excellent agreement with the measured velocity components as shown in Figs. 6 and 7.

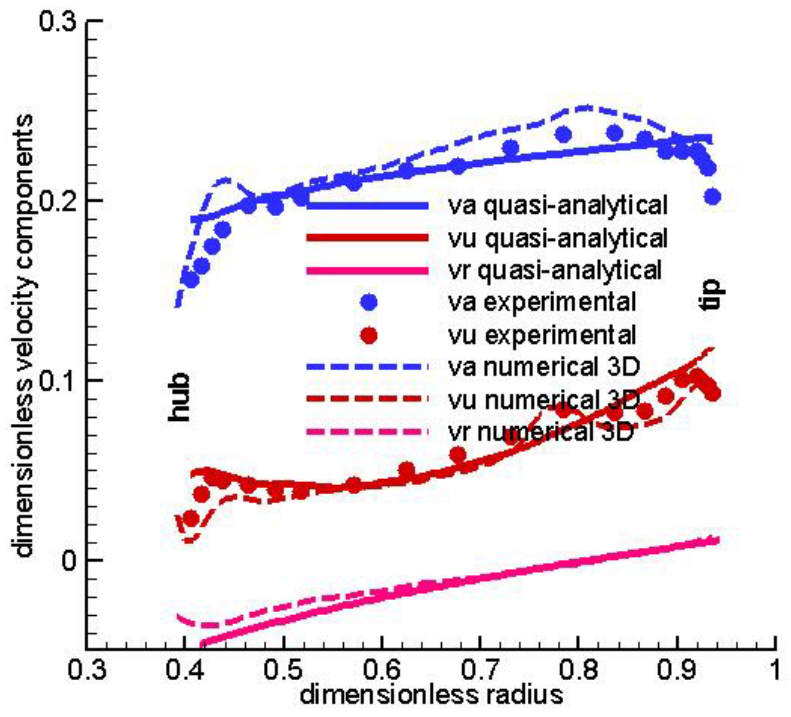

Figure 6. Axial, radial and circumferential velocity components at the BEP ( $\mathrm{T}$ case) operating point.

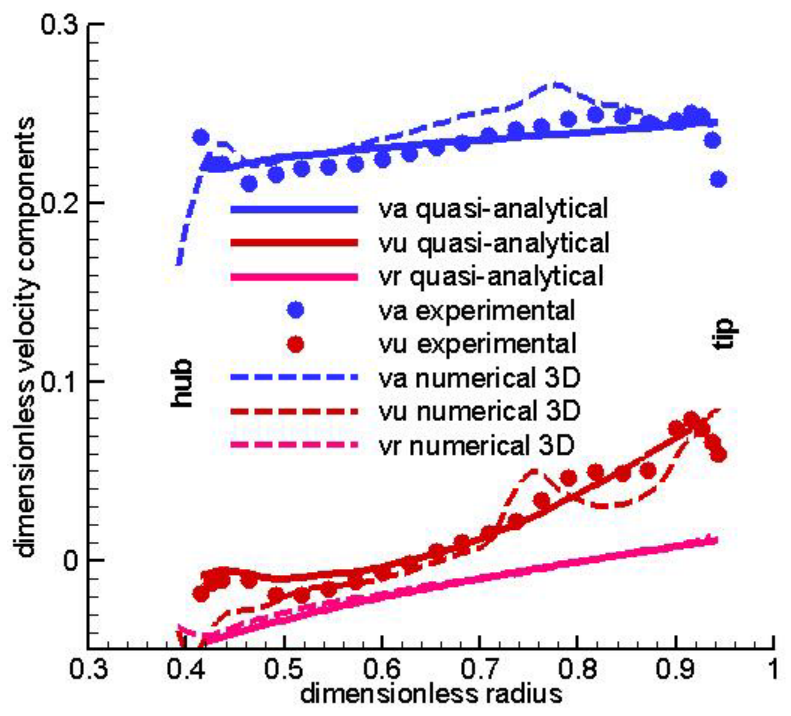

Figure 7. Axial, radial and circumferential velocity components at the FL (R case) operating point. 


\section{Conclusions}

The paper presents a quasi-analytical mathematical model for computing the swirling flow at the outlet of Kaplan runners, operated on a propeller curve with constant head and variable discharge. Compared to the initial version of the model [1], we introduce additional terms to account for radial velocity component and for the variable hub-to-tip rothalpy. The analytical expressions for these terms are assessed against numerical data from full three-dimensional simulations, since neither the radial velocity nor the rothalpy are available from measurements. Next, we use available experimental data to find so called swirl-free velocity profile, and to validate the computed results.

From practical point of view, the present model is intended for optimizing the swirl ingested by the draft tube, within an operating range, before actually designing the runner. In doing so, the model parameters are subject of optimization for the best draft tube response to the swirl emerging from the Kaplan runner.

\section{Acknowledgements}

Prof. Romeo Susan-Resiga and Dr. Sebastian Muntean were supported by Romanian Academy program "Swirling flow analysis and instabilities control in the turbomachineries". Tiberiu Ciocan was supported by the strategic grant POSDRU 107/1.5/S/77265 (2010) of the Ministry of Labor, Family and Social Protection, Romania, co-financed by the European Social Fund Investing in people.

Prof. Michel Cervantes and Dr. Håkan Nilsson are grateful to the Swedish Waterpower Centre (SVC) for the financial support. SVC has been established by the Swedish Energy Agency, Elforsk and Svenska Kraftnät together with Luleå University of Technology, The Royal Institute of Technology, Chalmers University of Technology and Uppsala University. (www.svc.nu)

\section{References}

[1] Susan-Resiga R F, Muntean S, Avellan F, and Anton I 2011 Mathematical modeling of swirling flow in hydraulic turbines for the full operating range, Appl. Math. Model., 35(10) pp. 47594773, doi:10.1016/j.apm.2011.03.052.

[2] Lugaresi A and Massa A 1988 Kaplan turbines: design trends in the last decade, Water Power and Dam Construction 40, pp. 12-17

[3] Radha Krishna H C (Editor) 1997 Hydraulic Design of Hydraulic Machinery, Avebury Publishing House.

[4] Anton I 1979 Hydraulic turbines, Facla Publishing House, Timisoara, Romania (in Romanian)

[5] Gebart B R, Gustavsson L H, and Karlsson R I (Editors) 2000 Proc. of Turbine-99 - Workshop on draft tube flow (Porjus, Sweden, 20-23 June 1999) Luleå University of Technology, Technical Report 2000:11

[6] Engström T F, Gustavsson L H, and Karlsson R I (Editors) 2001 Proc. of Turbine-99 Workshop 2, (Älvkarleby, Sweden, 18-20 June 2001) Luleå University of Technology.

[7] Cervantes M J, Engström T F, and Gustavsson L H (Editors) 2005 Proc. of Turbine-99 III. (Porjus, Sweden, 7-9 December 2005) Luleå University of Technology, Technical Report 2005:20.

[8] Andersson U 2009 An Experimental Study of the Flow in a Sharp-Heel Kaplan Draft Tube PhD Thesis (Luleå: Sweden)

[9] Wang S, Rusak Z 1997 The dynamics of the swirling flow in a pipe and transition to axisymmetric vortex breakdown, J. Fluid Mech., 340, pp. 177-223.

[10] Bergström J 1999 Approximations of numerical errors and boundary conditions in a draft tube. Proc. of Turbine 99 - Workshop on Draft Tube Flow (Porjus, Sweden, 2023 June 1999) eds B R Gebart et al.

[11] Cervantes M J and Gustavsson H 2007 On the use of the Squire-Long equation to estimate radial velocities in swirling flows, J. of Fluid Eng. - Trans. ASME, 129(2), pp. 209-217 